\title{
Proctalgia Fugax
}

National Cancer Institute

\section{Source}

National Cancer Institute. Proctalgia Fugax. NCI Thesaurus. Code C113615.

Recurrent episodes of pain localized to the anus or lower rectum which lasts seconds to minutes. There is no pain between episodes. 